\title{
Caught red-handed: uric acid is an agent of inflammation
}

\author{
Yan Shi \\ Immunology Research Group and Department of Microbiology \& Infectious Diseases, University of Calgary, Calgary, Alberta, Canada.
}

\begin{abstract}
Inflammation occurs in response to both pathogenic insult and tissue damage under sterile conditions, with the latter contributing to the pathogenesis of many diseases. Although several endogenous substances, including uric acid, have been suggested to alert the body to danger and to stimulate inflammation, little is known about their contribution to such responses in vivo. In this issue of the JCI, Kono et al. use newly generated mice with reduced levels of uric acid to investigate its role as an endogenous signal of tissue damage in inflammatory responses to hepatic injury. They find that uric acid is released from dying tissues and induces inflammation to cell death but is not involved in the response to microbial molecules or sterile irritant particles. I believe this to be the first report of an endogenous danger signal acting as a physiological regulator of inflammation.
\end{abstract}

PAMPs vs. DAMPs: evidence needed

Charles Janeway proposed the concept of innate immunity over 20 years ago, when he suggested that APCs expressed germline-encoded receptors that sensed microbial products (pathogen-associated molecular patterns [PAMPs]) and that triggering of these receptors initiated inflammatory and adaptive immune responses (1). Several years later, Polly Matzinger developed her danger hypothesis, whereby she proposed that APCs can sense endogenous signals released by damaged cells/tissues (signals she later termed damage-associated molecular patterns [DAMPs]) to trigger an immune response in the absence of PAMPs (i.e., under sterile conditions) (2). Generally, it is recognized that the two theories deal with two different sets of immune surveillance: external (exogenous) insults are detected by the innate immune system, while internal (endogenous) danger is the remit of Matzinger's hypothesis. The two concepts are symmetrical and complementary. However, their fates have turned out to be quite different. Janeway's hypothesis has been developed into the robust system of innate immune recognition, with many of the molecular intricacies of receptors, ligands, and signaling mechanisms described in the finest details (3). By contrast, the concept of endogenous adjuvants

Conflict of interest: The author has declared that no conflict of interest exists.

Citation for this article: J Clin Invest. 2010; 120(6):1809-1811. doi:10.1172/JCI43132. or danger signals is less well developed and, to a large extent, remains a conceptual discussion. Lack of elegance in the danger theory itself is certainly not the reason. Rather, the danger hypothesis thus far has not gathered the robust experimental support and verification that Janeway's proposal of innate immunity has relished.

Currently, there are several endogenous substances that are candidate DAMPs, including high-mobility group box 1 (HMGB1), heat shock proteins, and uric acid (4), and it is thought these are the substances released from injured cells that mediate inflammatory responses in the absence of PAMPs. While some of these have been found to stimulate sterile inflammatory responses in vitro, it has remained an open question as to whether any of them has a nonredundant role in a physiological/pathological situation. In this issue of the JCI, Kono et al. offer support for the idea that uric acid levels regulate sterile inflammation in vivo (5).

\section{An in vivo model of uric acid- induced sterile inflammation}

Uric acid is the final product of purine metabolism in humans and higher primates. It has been known for a long time to have a role in gout, since this painful inflammatory condition is the result of uric acid (monosodium urate [MSU]) crystals precipitating in joints and capillaries (6). Its association with other forms of inflammation and immune regulation was not apparent until it was found to serve as an adjuvant in CTL responses against particulate antigens (7). In the last several years, we have learned a number of features of this simple catabolite. First, it appears that uric acid has to be in the range of supersaturation $(>70 \mu \mathrm{g} / \mathrm{ml})$ to exert its adjuvant effect, implying that it elicits this effect as a crystal. Cell death results in local supersaturation of uric acid, as cytolysis generates large quantities of purines from RNA and DNA. Second, crystalline MSU binds to lipids on the surface of APCs and signals through the NACHT, LRR, and PYD domain-containing protein 3 (NLRP3) inflammasome to produce IL-1 $\beta(8,9)$. Third, preformed MSU crystals appear to promote tumor rejection (10). Fourth, depletion of uric acid reduces the effect of alum in vaccines (11). Despite these findings, which hinted at the ability of uric acid to act as a DAMP, to systematically study the effect of uric acid, an animal model is needed, as pharmacological inhibition of uric acid is short lived. Furthermore, uricase-deficient mice die from kidney failure before weaning, unless rescued by injection of the enzyme conjugated to polyethylene glycol (12). The work of Kono et al. (5) therefore introduces what I believe to be the first viable animal models generated for the purpose of immunological research on uric acid. Using the mice they generated, they confirmed that uric acid can participate in the induction of sterile inflammation in vivo.

Kono et al. (5) approached the issue of how to modulate uric acid levels from the opposite direction of uricase deficiency, reducing uric acid levels by introducing transgenes to increase uricase expression. Several details of their design are worth noting. In mammals, uricase is expressed only in the liver. In this study, one version of the transgene is fused to a viral signal sequence that promotes extracellular secretion, increasing its availability in interstitial fluids. The other construct targets uricase to peroxisomes, where it reduces intracellular pools of uric acid, and its effects are only apparent upon cytolysis. Both transgenes are under the control 


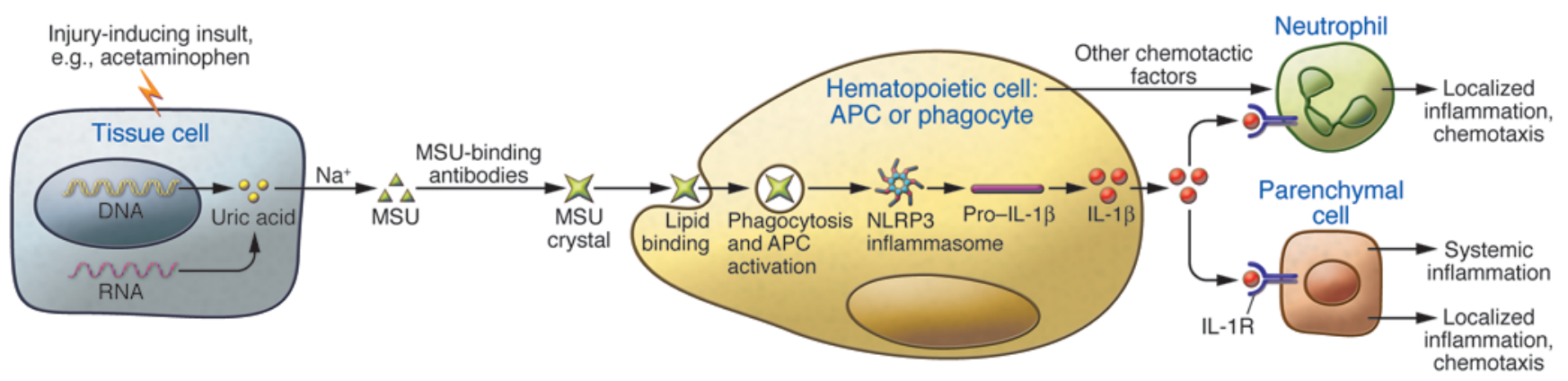

Figure 1

Proposed mechanism by which uric acid triggers inflammation. Tissue damage of various forms produces and releases uric acid from purines via xanthine oxidase. Uric acid in the extracellular environment binds to sodium to form MSU. With assistance from serum factors, such as MSU-binding antibodies, MSU crystals precipitate. The crystals engage APC membrane lipids to trigger phagocytosis and APC activation. MSU gains entry into the cell where it interacts with the NLRP3 inflammasome to produce IL-1 $\beta$. IL-1 $\beta$ signals via its receptor (IL-1R) on parenchymal cells, and along with other chemotactic factors, it serves as a beacon to attract neutrophils and other immune cells, thereby eliciting an inflammatory response to tissue injury.

of the actin promoter to maximize tissue distribution. The authors first focus on acetaminophen-induced hepatotoxicity, a model of the most common cause of acute liver failure in the Western world, and study the role of uric acid levels in the associated inflammation (5). Their results reveal a 2-step process: acute cell death followed by neutrophil infiltration/inflammation. The first step is shown not to be linked to uric acid, as markers of liver cell damage are not affected by reduced uric acid levels. However, the tissue response to cell death is shown to be by and large driven by uric acid, as neutrophil infiltration and myeloperoxidase production are reduced in the transgenic mice. The authors obtain similar results when killed cells are injected as a source of tissue injury. Consistent with previous reports from this group (13-15), uric acid plays a role where it is made available in sufficient quantities to promote IL- $1 \beta$ production. Interestingly, seemingly similar responses to sterile crystals of silica, zymosan, and LPS are not reduced in the uric acid-depleted mice and, in fact, are increased. Thus, it seems that these substances can trigger inflammation on their own, whereas cell death must use uric acid as the messenger to gain "attention."

\section{The devil is in the detail}

The authors profess amazement that despite many proposed substances, depletion of uric acid induces a strong protective effect and reduced inflammation in the context of cell death (5). This suggests that uric acid, at least in this context, may be the major trigger of inflammation. If that is the case, we have had a good head start because uric acid-induced inflammatory signals are being elucidated in detail, by this and several other groups. Together, a general picture of uric acid signaling and effector function is coming into focus (Figure 1). The association with NLRP3 inflammasome-mediated IL- $1 \beta$ production appears to be at the center of its systematic inflammatory effect and chemotaxis. Uric acid activates the NLRP3/ASC/caspase-1 complex, leading to the conversion of pro-IL- $1 \beta$ into its secreted, active form, similar to other inflammatory substances (8). One crucial detail is illustrated by 3 previous papers from this group (13-15). Uric acid drives IL-1 $\beta$ production from hematopoietic cells, although activation of these cells themselves is not driven by IL- $1 \beta$, as they are the initial responders to the original etiological insults, such as drug- or injury-induced tissue damage. The secreted IL- $1 \beta$ serves as a systemic signal for inflammation, signaling via its receptor (IL-1R) in the peripheral tissues. The recent papers by this group define the boundary among localized "danger" sensing, the activation of APCs, and the fully fledged inflammatory response and possibly suggest how uric acid works as an immune adjuvant $(5,13-15)$. This point is further reinforced by the observation of Kono et al. that uric acid does not participate in the initial acute cytotoxic event; rather, neutrophils heed the call of uric acid released from the dying cells, leading to amplified inflammation (5).

\section{Not so trivial trivia}

The work of Kono et al. (5), along with the group's previous reports (13-15), may have inadvertently established a connection with some clinical reports over the years. It has long been known that high uric acid levels in patients are predictive of cardiovascular pathologies (16). Such a connection has been an etiological dilemma. Uric acid is an antioxidant, a major recipient of reactive oxygen species. The enhanced inflammation to sterile irritants other than cell death (e.g., silica crystals) in the uric acid-depleted mice reported in this paper (5) may offer a glimpse into a protective role for this metabolite in other conditions. One can imagine that sufficient uric acid to induce endogenous inflammatory responses only occurs with large-scale cell death and must be associated with uric acid precipitation; in other words, a general antioxidant is converted into a strong inflammatory stimulant only upon its phase transition (6). Although the work of Kono et al. did not address this specific issue (5), recent data have suggested that serum antibodies against uric acid may facilitate its crystallization (17).

Purine metabolism is an aspect of intrigue in biology. Lower species, such as microbes, process it to the end product of ammonia. Through evolution, its metabolism becomes less and less complete. In mammals, uric acid is processed to become allantoin and uricase expression is confined to peroxisomes in hepatocytes. However, in primates, a silent mutation has eliminated the uricase function, and gout ensues. It has been an interesting discussion as to why such a loss of function was necessary at the expense of a major disease and possibly cardiovascular complications. Two hypotheses have been put forward. One proposal is that loss of 
uric acid degradation sustains serum salt levels and sufficiently high blood pressure in low salt environments (18). The other, more blithe, idea is that uric acid targets purine receptors in the cerebral cortex in ways similar to caffeine, rendering primates more mentally alert and intellectuals more prone to gouty arthritis (the disease of distinction) $(19,20)$. In light of the work of Kono et al. (5), we have gained experimental evidence that evolution may have had an additional incentive for eliminating the ability to degrade uric acid. In conjunction with a urate anion exchange regulator in the kidney (21), elevated uric acid levels push to the edge of precipitation. As such, any cell death or tissue insult can immediately raise levels above those required for precipitation $(70 \mu \mathrm{g} / \mathrm{ml})$, thereby bringing into the picture a powerful trigger of inflammation, MSU crystals. By lacking the ability to degrade uric acid, the primate host therefore becomes more efficient at monitoring cellular stress. In large-scale injury and trauma, as well as other situations where inflammation is collectively beneficial to the host, high uric acid could have been a substantial survival advantage (22). If future research supports such reasoning, it is expected that uric acid will be found to participate in sterile inflammation in additional contexts and that it will present the promise of a therapeutic target.

\section{Acknowledgments}

The author is funded by the NIH, the Canadian Institutes of Health Research, Canada Research Chair program, and the Alberta Heritage Foundation for Medical Research.

Address correspondence to: Yan Shi, 4A18 HRIC, Faculty of Medicine, University of Calgary, 3330 Hospital Dr. NW, Calgary, Alberta, Canada T2N 4N1. Phone: 403.220.2536; Fax: 403.220.2772; E-mail: yshi@ucalgary.ca.

1. Janeway CA Jr. Approaching the asymptote? Evolution and revolution in immunology. Cold Spring Harb Symp Quant Biol. 1989;54 pt 1:1-13.

2. Matzinger P. Tolerance, danger, and the extended family. Annu Rev Immunol. 1994;12:991-1045.

3. Medzhitov R. Approaching the asymptote: 20 years later. Immunity. 2009;30(6):766-775.

4. Foell D, Wittkowski H, Roth J. Mechanisms of disease: a 'DAMP' view of inflammatory arthritis. Nat Clin Pract Rheumatol. 2007;3(7):382-390.

5. Kono H, Chen C-J, Ontiveros F, Rock KL. Uric acid promotes an acute inflammatory response to sterile cell death in mice. J Clin Invest. 2010; 120(6):1939-1949.

6. Shi Y, Mucsi AD, Ng G. Monosodium urate crystals in inflammation and immunity. Immunol Rev. 2010;233(1):203-217.

7. Shi Y, Evans JE, Rock KL. Molecular identification of a danger signal that alerts the immune system to dying cells. Nature. 2003;425(6957):516-521.

8. Martinon F, Petrilli V, Mayor A, Tardivel A, Tschopp J. Gout-associated uric acid crystals activate the NALP3 inflammasome. Nature. 2006; 440(7081):237-241.

9. Ng G, et al. Receptor-independent, direct membrane binding leads to cell-surface lipid sorting and Syk kinase activation in dendritic cells. Immunity. 2008;29(5):807-818.

10. Hu DE, Moore AM, Thomsen LL, Brindle KM. Uric acid promotes tumor immune rejection. Cancer Res. 2004;64(15):5059-5062.

11. Kool M, et al. Alum adjuvant boosts adaptive immunity by inducing uric acid and activating inflammatory dendritic cells. J Exp Med. 2008; 205(4):869-882.

12. Wu X, et al. Hyperuricemia and urate nephropathy in urate oxidase-deficient mice. Proc Natl Acad Sci US A. 1994;91(2):742-746.

13. Chen CJ, Kono H, Golenbock D, Reed G, Akira S, Rock KL. Identification of a key pathway required for the sterile inflammatory response triggered by dying cells. Nat Med. 2007;13(7):851-856.

14. Chen CJ, et al. MyD88-dependent IL-1 receptor signaling is essential for gouty inflammation stimulated by monosodium urate crystals. J Clin Invest. 2006;116(8):2262-2271.

15. Kono H, Karmarkar D, Iwakura Y, Rock KL. Identification of the cellular sensor that stimulates the inflammatory response to sterile cell death. J Immunol. 2010;184(8):4470-4478.

16. Feig DI, Kang DH, Johnson RJ. Uric acid and cardiovascular risk. NEnglJ Med. 2008;359(17):1811-1821.

17. Kanevets U, Sharma K, Dresser K, Shi Y. A role of IgM antibodies in monosodium urate crystal formation and associated adjuvanticity. J Immunol. 2009;182(4):1912-1918.

18. Watanabe $\mathrm{S}$, et al. Uric acid, hominoid evolution, and the pathogenesis of salt-sensitivity. Hypertension. 2002;40(3):355-360.

19. Montoye HJ, Mikkelsen WM. Serum uric acid and achievement in high school. Arthritis Rheum. 1973;16(3):359-362.

20. Sofaer JA, Emery AE. Genes for super-intelligence? JMed Genet. 1981;18(6):410-413.

21. Enomoto A, et al. Molecular identification of a renal urate anion exchanger that regulates blood urate levels. Nature. 2002;417(6887):447-452.

22. Nathan C. Points of control in inflammation. Nature. 2002;420(6917):846-852. 